\title{
Chronic pulmonary aspergillosis commonly complicates treated pulmonary tuberculosis with residual cavitation
}

\author{
lain D. Page (10 ${ }^{1,2}$, Rosemary Byanyima ${ }^{3}$, Sharath Hosmane ${ }^{4}$, Nathan Onyachi ${ }^{5}$, \\ Cyprian Opira ${ }^{6}$, Malcolm Richardson ${ }^{1,7}$, Richard Sawyer ${ }^{4}$, Anna Sharman ${ }^{4}$ and \\ David W. Denning ${ }^{1,2}$
}

\begin{abstract}
Affiliations: 'Division of Infection, Immunity and Respiratory Medicine, Faculty of Biology, Medicine and Health, The University of Manchester, Manchester Academic Health Science Centre, Manchester, UK. ${ }^{2}$ National Aspergillosis Centre, ECMM Excellence Centre of Medical Mycology, Manchester University NHS Foundation Trust, Manchester, UK. ${ }^{3}$ Kampala Imaging Centre, Kampala, Uganda. ${ }^{4}$ Radiology Dept, Manchester University NHS Foundation Trust, Manchester, UK. ${ }^{5}$ Gulu Regional Referral Hospital, Gulu, Uganda. ${ }^{6}$ St Mary's Hospital at Lacor, Gulu, Uganda. ${ }^{7}$ Mycology Reference Centre, ECMM Excellence Centre of Medical Mycology, Manchester University NHS Foundation Trust, Manchester, UK.
\end{abstract}

Correspondence: lain D. Page, National Aspergillosis Centre, 2nd Floor, Education and Research Centre, Wythenshawe Hospital, Southmoor Road, Manchester, M23 9LT, UK. E-mail: iain.pageamanchester.ac.uk

@ERSpublications

Chronic pulmonary aspergillosis complicates tuberculosis frequently enough to represent a global public health issue http://ow.ly/sYRO30nhg8t

Cite this article as: Page ID, Byanyima R, Hosmane S, et al. Chronic pulmonary aspergillosis commonly complicates treated pulmonary tuberculosis with residual cavitation. Eur Respir J 2019; 53: 1801184 [https://doi.org/10.1183/13993003.01184-2018].

ABSTRACT Chronic pulmonary aspergillosis (CPA) complicates treated pulmonary tuberculosis (TB), with high 5-year mortality. We measured CPA prevalence in this group.

398 Ugandans with treated pulmonary TB underwent clinical assessment, chest radiography and Aspergillus-specific IgG measurement. 285 were resurveyed 2 years later, including computed tomography of the thorax in 73 with suspected CPA. CPA was diagnosed in patients without active TB who had raised Aspergillus-specific IgG, radiological features of CPA and chronic cough or haemoptysis.

Author-defined CPA was present in 14 (4.9\%, 95\% CI 2.8-7.9\%) resurvey patients. CPA was significantly more common in those with chest radiography cavitation $(26 \%$ versus $0.8 \%$; $\mathrm{p}<0.001)$, but possibly less frequent in HIV co-infected patients ( $3 \%$ versus $6.7 \%$; $=0.177$ ). The annual rate of new CPA development between surveys was $6.5 \%$ in those with chest radiography cavitation and $0.2 \%$ in those without $(p<0.001)$. Absence of cavitation and pleural thickening on chest radiography had $100 \%$ negative predictive value for CPA. The combination of raised Aspergillus-specific IgG, chronic cough or haemoptysis and chest radiography cavitation had $85.7 \%$ sensitivity and $99.6 \%$ specificity for CPA diagnosis.

CPA commonly complicates treated pulmonary TB with residual chest radiography cavitation. Chest radiography alone can exclude CPA. Addition of serology can diagnose CPA with reasonable accuracy.

This article has supplementary material available from erj.ersjournals.com

Received: May 082018 | Accepted after revision: Dec 192018

Copyright $\odot$ ERS 2019. This article is open access and distributed under the terms of the Creative Commons Attribution Non-Commercial Licence 4.0. 


\section{Introduction}

Tuberculosis (TB) affected an estimated 10.4 million people in 2016, with 1.3 million attributable deaths in those without HIV and a further 374000 deaths in HIV co-infected persons [1]. Just 57\% of pulmonary TB cases reported to the World Health Organization (WHO) were bacteriologically confirmed. Chronic pulmonary aspergillosis (CPA) both complicates and mimics treated pulmonary $\mathrm{TB}[2,3]$. The prevalence of $\mathrm{CPA}$ in patients with treated $\mathrm{TB}$ and the contribution of misdiagnosed $\mathrm{CPA}$ to $\mathrm{TB}$ prevalence estimates are unclear.

The European Respiratory Society (ERS) published the first guidelines on CPA diagnosis and management in 2016 [4]. Chronic cavitary pulmonary aspergillosis (CCPA) is the most common form of CPA [5, 6]. It presents with progressive cavitation and symptoms including weight loss, fatigue, cough, breathlessness, chest discomfort and haemoptysis. The 5-year mortality of CCPA is $38-80 \%[3,7]$. However, surgery is curative in selected patients with localised disease and has been safely delivered in resource-poor settings [8-11]. Treatment with oral azole drugs can prevent clinical and radiological progression [12-14]. Chronic fibrosing pulmonary aspergillosis (CFPA) complicates untreated CCPA, with destruction of the lung [15]. Figure 1 illustrates the subtypes of CPA.

Sputum culture for Aspergillus is often negative in CPA [2]. Measurement of Aspergillus-specific IgG is central to CPA diagnosis $[4,16]$, but until recently had been inadequately validated for use in this context [17]. Both tests are infrequently available in areas of high TB prevalence [18].

Our recent studies demonstrate that Siemens Immulite Aspergillus-specific IgG is the best performing commercial assay for CPA diagnosis [5]. It has a sensitivity of $93.4 \%$ for UK CPA cases and specificity of 98.7\% against healthy controls or $97.9 \%$ against Ugandan controls with treated pulmonary TB at a cut-off of $20 \mathrm{mg} \cdot \mathrm{L}^{-1}$, regardless of HIV status [19].

The global prevalence of CPA secondary to TB has been estimated at 1.2 million cases [20]. CPA has a global distribution, including over 200 reported cases in Africa [2, 3, 8, 11, 12, 21-26]. Prevalence estimates are based on the results of a 1968-1970 survey describing aspergilloma in 20\% of 544 British patients with residual lung cavities after TB treatment [27]. That study was performed prior to computed tomography (CT) scanning and the HIV pandemic. Subsequent studies have demonstrated that aspergilloma is only present in a third of CPA cases [2].

We aimed to undertake the first accurate measurement of CPA prevalence. We conducted a community-based, cross-sectional prospective survey with systematic CT scanning of the thorax in an area of high TB prevalence. The prevalence of CPA in patients with and without HIV infection and chest radiography cavitation was compared, and the annual rate of CPA development between two surveys 2 years apart described.

Current CPA diagnostic criteria are arbitrary and based on expert opinion [4]. We aimed to conduct the first assessment of associations between raised Aspergillus-specific IgG and individual clinical or radiological features.

FIGURE 1 Venn diagram showing subtypes of chronic pulmonary aspergillosis (CPA). CCPA: chronic cavitary pulmonary aspergillosis; CFPA: chronic fibrosing pulmonary aspergillosis; AN: Aspergillus nodules; SA: simple aspergilloma. All patients have CPA. The size of each group represents the approximate estimated prevalence of each condition. Patients with subacute invasive pulmonary aspergillosis do not have CPA and are not included.

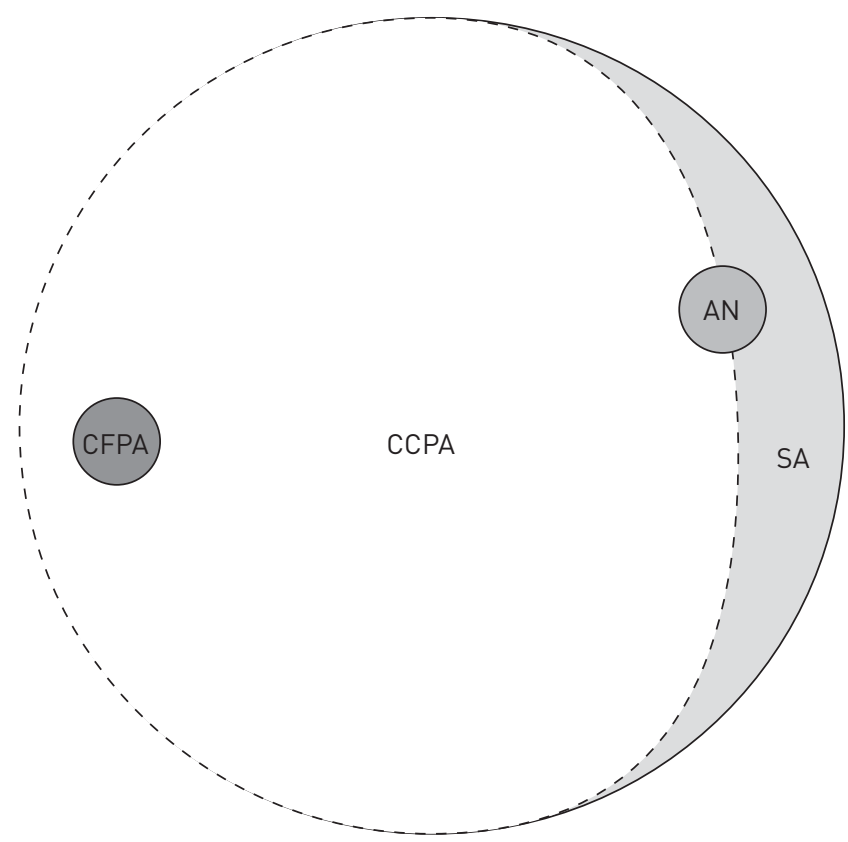


Pulmonary TB mostly occurs in resource-poor settings, where access to CT scanning of the thorax and Aspergillus-specific IgG testing is limited $[18,20]$. We describe the accuracy of CPA diagnosis by chest radiography and clinical assessment, with or without serology, in comparison to CT-confirmed disease.

\section{Methods}

Adults aged $\geqslant 16$ years, who were in the final month of pulmonary TB treatment or completed treatment in 2005 or later, were recruited in Gulu (Uganda) from October 2012 to January 2013. Patients were recruited from Gulu Regional Referral Hospital TB and HIV clinics or by Gulu District Health Office staff. Eligible patients had smear-positive TB or complete resolution of symptoms on therapy. We aimed to recruit $50 \%$ of patients with HIV co-infection at the time of TB treatment. Patients with no recorded HIV status who declined testing were ineligible.

Patients underwent structured clinical assessment. "Severe dyspnoea" represents a Medical Research Council dyspnoea score of 4 or 5. Chest radiography was performed at St Mary's Hospital at Lacor, Gulu. Serum was stored at $-80^{\circ} \mathrm{C}$ at the Joint Clinical and Research Centre (JCRC) in Gulu. Immulite 2000 Aspergillus fumigatus-specific IgG testing (Siemens, Berlin, Germany) was performed at Christie Hospital (Manchester, UK) in July 2014. Raised levels are $\geqslant 20 \mathrm{mg} \cdot \mathrm{L}^{-1}$. CD4 testing was performed by FACSCalibur flow cytometry (Becton Dickinson, Franklin Lakes, NJ, USA) at the JCRC, if no recent result was documented (<6 months).

Patients were traced and reassessed (resurvey) between October 2014 and January 2015, with repeat chest radiography and Aspergillus-specific IgG. GeneXpert IV Mycobacterium tuberculosis nucleic acid amplification testing (Cepheid, Sunnyvale, CA, USA) was performed at Gulu Regional Referral Hospital for all patients who could provide a sputum sample. CT scanning of the thorax was performed using a Dual Slice scanner (GE, Boston, MA, USA) at the Kampala Imaging Centre (Uganda) on those with Aspergillus-specific IgG $>10 \mathrm{mg} \cdot \mathrm{L}^{-1}$ or report of a fungal ball on chest radiography by any radiologist in the initial survey. Digital CT images were reported using Osirix X software (Pixmeo, Bernex, Switzerland). Two radiologists, blinded to clinical and serological findings, provided radiological reports. The senior respiratory radiologist (R.S.) at the UK National Aspergillosis Centre (Manchester, UK) provided a decisive report where initial reports differed.

In the first survey, probable CCPA was diagnosed when three criteria were met: 1) cough or haemoptysis for $\geqslant 1$ month, 2) cavitation or a fungal ball on chest radiography and 3) raised Aspergillus-specific IgG. Probable simple aspergilloma was diagnosed in patients with a fungal ball on chest radiography and raised Aspergillus-specific IgG, but no chronic cough or haemoptysis. All "probable CPA" is the combination of patients with probable CCPA or probable simple aspergilloma.

CPA was definitively diagnosed after the resurvey. A modified version of the DenNING et al. [21] 2003 criteria was used. CCPA cases had all of: 1) cough or haemoptysis for $\geqslant 1$ month, 2) raised Aspergillus-specific IgG, 3) absence of positive GeneXpert test for M. tuberculosis and 4) either paracavitary fibrosis or a fungal ball on CT of the thorax or progressive cavitation (either new cavitation or deterioration of pre-existing cavitation) on serial chest radiographs.

CFPA was diagnosed in patients with clinical and serological features of CCPA and complete destruction of at least two lobes of a lung on CT of the thorax. Simple aspergilloma was diagnosed in patients with a fungal ball on CT of the thorax and raised Aspergillus-specific IgG, but no cough or haemoptysis. The combined totals of CCPA, CFPA and simple aspergilloma are presented as "all CPA". A seronegative fungal ball was reported in patients with a fungal ball on CT of the thorax, but normal Aspergillus-specific IgG.

New CPA, developing during the 2-year follow-up period, was diagnosed in those who had normal Aspergillus-specific IgG in the initial survey, but CPA on resurvey, on the basis of resurvey criteria where CT scanning was performed and original survey criteria where it was not. Those patients diagnosed with $\mathrm{CPA}$ in the absence of CT of the thorax were reported as "probable CPA" in the final prevalence results.

\section{Ethical approval}

Ethical approval was granted by The University of Manchester Research Ethics Committee (7/6/12-11424), Gulu University Faculty of Medicine Institutional Review Board (GU/IRC/04/07/12), and the Uganda National Council for Science and Technology (HS 1253).

\section{Statistical analysis}

Statistical analysis was performed using SPSS version 20 (IBM, Armonk, NY, USA). The study was designed to measure the prevalence of CPA with an accuracy of $\pm 2.3 \%$ based on a predicted prevalence of $6 \%$. It had $80 \%$ power to detect an absolute difference of $9 \%$ in CPA prevalence between HIV-positive and 
HIV-negative groups with 5\% significance. Frequencies of categorical variables were compared by Fisher's exact test throughout, unless otherwise stated. Mean results were compared by the t-test and median results by the Mann-Whitney U-test. 95\% confidence intervals for disease prevalence were calculated by the Jeffreys method.

In additional post hoc analyses we assessed associations between potential clinical and environmental risk factors and CPA diagnosis or raised Aspergillus-specific IgG. We also assessed associations between individual symptoms and radiological findings and CPA diagnosis or raised Aspergillus-specific IgG. Odds ratios for associations between clinical or radiological features and CPA were calculated by bimodal regression analysis.

We calculated the sensitivity, specificity, and positive and negative predictive values of various combinations of symptoms, radiological results and Aspergillus-specific IgG testing in relation to final CPA diagnosis after CT of the thorax. The accuracy of chest radiography for diagnosis of CT-proven pathology was described in the same manner.

\section{Results}

Patient characteristics and initial survey results are shown in table 1. Median time from completion of TB treatment to recruitment was 29 months. Low CD4 counts were uncommon in HIV-positive patients. Cough and cavitation on chest radiography were more common in HIV-negative patients in the initial survey. $11(2.8 \%)$ patients met the criteria for probable CPA, comprising nine (2.3\%) with CCPA and two $(0.5 \%)$ with simple aspergilloma.

TABLE 1 Patient characteristics and initial survey results (2012)

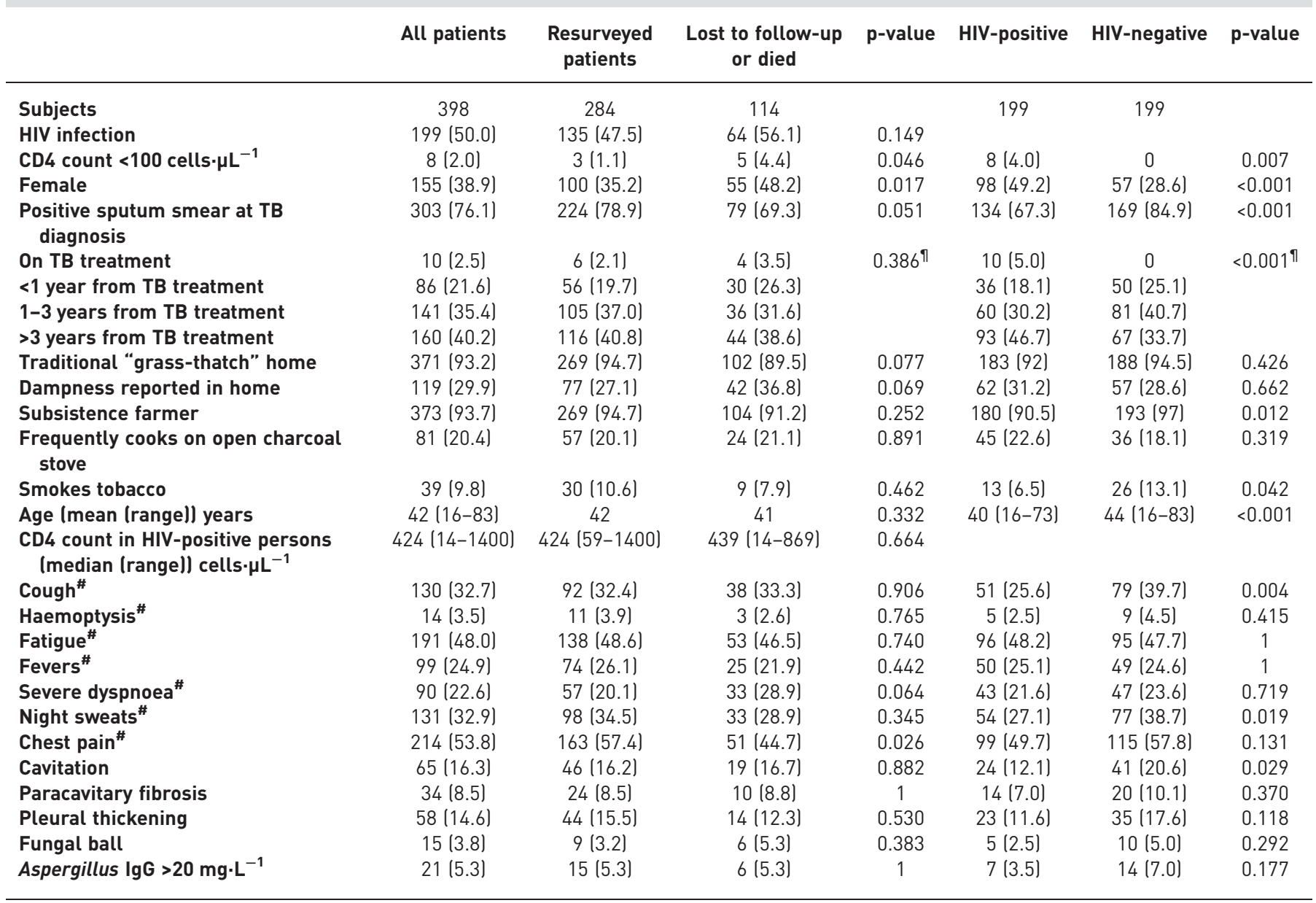

Data are presented as $\mathrm{n}$ or $\mathrm{n}(\%)$, unless otherwise stated. TB: tuberculosis. ${ }^{\#}$ : present for $\geqslant 1$ month; " : two-sided Chi-squared test. $\mathrm{p}$-values calculated by Fisher's exact test, except where indicated. Mean results compared by the t-test and median results by the Mann-Whitney U-test. $p<0.05$ considered significant. 
At the 2-year follow-up, 285 (71.6\%) patients were resurveyed and 19 (4.8\%) were confirmed to have died between surveys. $94(23.6 \%)$ could not be traced. Rates of loss to follow-up were similar in those with or without probable CPA $(27.3 \%$ versus $24.0 \%$; $=0.731)$. No patient with probable CPA died between surveys.

Results for resurvey patients are compared in table 2. While overall symptom frequency fell between surveys, frequency of haemoptysis and raised Aspergillus-specific IgG increased. Radiological findings consistent with CPA and elevated Aspergillus-specific IgG were more common in males $(\mathrm{p}=0.012-0.041)$, but symptoms consistent with CPA were not $(\mathrm{p}=0.270-0.830)$ (supplementary table $\mathrm{S} 1$ ).

CT of the thorax was performed in $73(25.6 \%)$ resurvey patients, including 32 out of 46 (69.6\%) with chest radiography cavitation in the original survey. CT of the thorax results and accuracy of chest radiography for identification of CT-proven pathology are shown in table 3. Two (0.7\%) resurvey patients had a positive GeneXpert test out of 26 samples tested. Neither met diagnostic criteria for CPA.

Definite CPA was diagnosed in 14 (4.9\%) resurvey patients. Full prevalence results are shown in table 4 . Two patients had a seronegative fungal ball. Another two who did not undergo CT of the thorax developed probable CPA between surveys, comprising one with a new fungal ball on chest radiography and one with chest radiography cavitation, plus progressive paracavitary fibrosis and pleural thickening and new haemoptysis. In total, 18 (6.3\%) resurvey cases had definite or probable CPA. There were an additional eight $(2.8 \%)$ patients with radiological features of CPA, other than a fungal ball and characteristic symptoms, but negative Aspergillus-specific IgG.

CPA was present in 12 out of $46(26.1 \%)$ resurvey patients with chest radiography cavities and two out of $239(0.8 \%)$ patients with no such cavities $(\mathrm{p}<0.001) .12(85.7 \%)$ of the 14 CPA cases had chest radiography cavities, all present in both surveys. Fungal ball was identified in nine (64.3\%) CPA cases. Two (14.3\%) CPA cases without a fungal ball had destroyed lung and one (7.1\%) had progressive cavitation. Two (14.3\%) CPA

TABLE 2 Results for resurvey patients

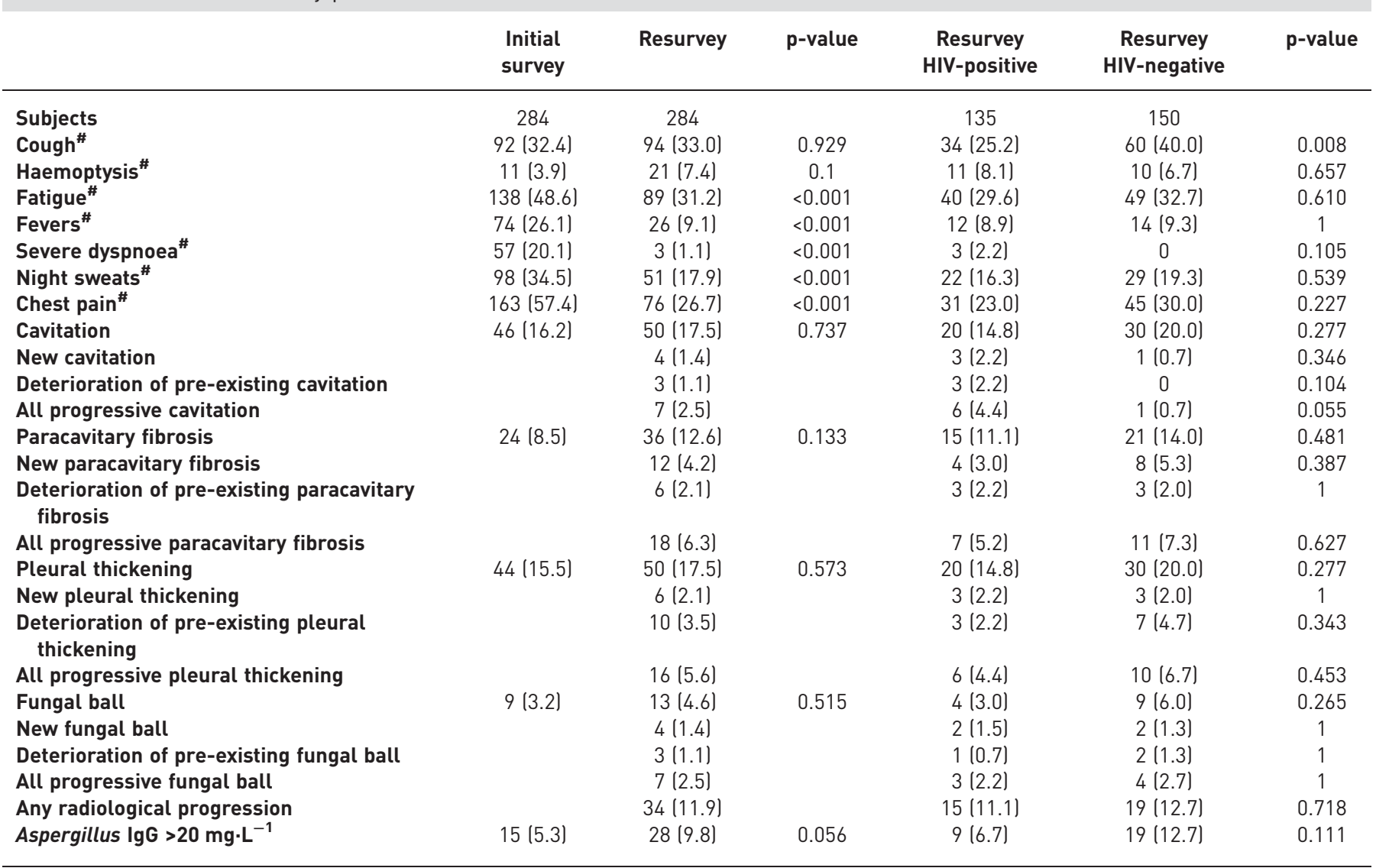

Data are presented as $\mathrm{n}$ or $\mathrm{n}(\%)$, unless otherwise stated. ${ }^{*}$ : present for $\geqslant 1$ month. All $\mathrm{p}$-values calculated by Fisher's exact test. $\mathrm{p}<0.05$ considered significant. 
TABLE 3 Radiology results for the computed tomography (CT) of the thorax group

\begin{tabular}{|c|c|c|c|c|c|c|c|c|c|c|}
\hline CT: radiologist & $\begin{array}{c}\text { CT: radiologist } \\
2\end{array}$ & $\begin{array}{c}\text { Final CT } \\
\text { result }\end{array}$ & HIV-positive & HIV-negative & $p$-value & $\begin{array}{l}\text { Chest radiography } \\
\text { result }\end{array}$ & $\begin{array}{c}\text { Sensitivity } \\
\%\end{array}$ & $\begin{array}{c}\text { Specificity } \\
\%\end{array}$ & $\begin{array}{l}\mathrm{PPV}^{\#} \\
\%\end{array}$ & $\begin{array}{c}\text { NPV }^{\#} \\
\%\end{array}$ \\
\hline 73 & 73 & 73 & 26 & 47 & & 73 & & & & \\
\hline $12(16.4)$ & $7(9.6)$ & $11(15.1)$ & $3(11.5)$ & $8(17)$ & 0.736 & 9 (12.3) & 36.4 & 91.9 & 44.4 & 89.1 \\
\hline $41(56.2)$ & $32(43.8)$ & 35 (47.9) & $9(34.6)$ & 26 (55.3) & 0.142 & $32(43.8)$ & 77.1 & 86.8 & 84.4 & 80.5 \\
\hline $24(32.9)$ & 30 (41.1) & 28 (38.4) & $6(23.1)$ & 22 (46.8) & 0.077 & 20 (27.4) & 50 & 86.7 & 70 & 73.6 \\
\hline $49(67.1)$ & 64 (87.7) & 48 (65.8) & 14 (53.8) & 34 (72.3) & 0.129 & 31 (42.5) & 60.4 & 92 & 93.5 & 54.8 \\
\hline 68 (93.2) & 58 (79.5) & 62 (84.9) & 20 (76.9) & 42 (89.4) & 0.183 & & & & & \\
\hline 64 (87.7) & 44 (60.3) & 54 (74.0) & 16 (61.5) & 38 (80.9) & 0.096 & & & & & \\
\hline $3(4.1)$ & $3(4.1)$ & $3(4.1)$ & $1(3.8)$ & $2(4.3)$ & 1 & & & & & \\
\hline
\end{tabular}
for chest radiography. ${ }^{\#}$ : of chest radiography for identification of CT of the thorax findings. All p-values calculated by Fisher's exact test. $p<0.05$ considered significant. 


\begin{tabular}{lcccc}
\multicolumn{4}{l}{ TABLE 4 Frequency of chronic pulmonary aspergillosis (CPA) } & \\
& All patients & HIV-positive & HIV-negative & p-value \\
\hline Subjects & 285 & 135 & 150 & \\
CCPA & $10(3.5(1.8-6.1))$ & $2(1.5(0.3-4.7))$ & $8(5.3(2.6-9.8))$ & 0.108 \\
CFPA & $3(1.1(0.3-2.8))$ & $1(0.7(0.1-3.4))$ & $2(1.3(0.3-4.2))$ & 1 \\
Simple aspergilloma & $1(0.4(0-1.6))$ & $1(0.7(0.1-3.4))$ & $0(0(0-1.7))$ & 0.474 \\
All definite CPA & $14(4.9(2.8-7.9))$ & $4(3.0(1-6.9))$ & $10(6.7(3.5-11.5))$ & 0.177 \\
Seronegative fungal ball & $2(0.7(0.1-2.2))$ & $1(0.7(0.1-3.4))$ & $1(0.7(0.1-3.1))$ & 1 \\
Probable CPA in non-CT group & $2(0.7(0.1-2.2))$ & $2(1.5(0.3-4.7))$ & $0(0(0-1.7))$ & 0.223 \\
All definite and probable CPA & $18(6.3(3.9-9.6))$ & $7(5.2(2.3-9.9))$ & $11(7.3(4-12.3))$ & 0.478
\end{tabular}

Data are presented as $\mathrm{n}$ or $\mathrm{n}(\%(95 \% \mathrm{CI}))$, unless otherwise stated. CCPA: chronic cavitary pulmonary aspergillosis; CFPA: chronic fibrosing pulmonary aspergillosis; CT: computed tomography. All p-values calculated by Fisher's exact test. $p<0.05$ considered significant.

cases met radiological case criteria for CPA due to paracavitary fibrosis alone. Both also had pleural thickening (figure 2). Eight (57.1\%) CPA cases had progressive chest radiography changes.

All eight resurvey patients with probable CPA in the original survey met CT of the thorax criteria for CPA on resurvey.

Seven (2.5\%) resurvey patients developed CPA between surveys, comprising five (1.8\%) diagnosed by CT of the thorax and two (0.7\%) diagnosed only by chest radiography criteria. Six (86\%) of the new CPA cases had chest radiography cavitation on initial survey. The annual rate of CPA development was $1.25 \%$ in all patients, $6.5 \%$ in the 46 resurvey patients who had chest radiography cavitation on initial survey and $0.2 \%$ in the 239 resurvey patients who did not $(\mathrm{p}<0.001)$. The incidence of new CPA was similar in all "time from TB therapy" groups. Five $(71.4 \%)$ of those who developed new CPA did so within 5 years of completion of TB therapy. Chest radiography progression in a new case of CPA is shown in figure 3.
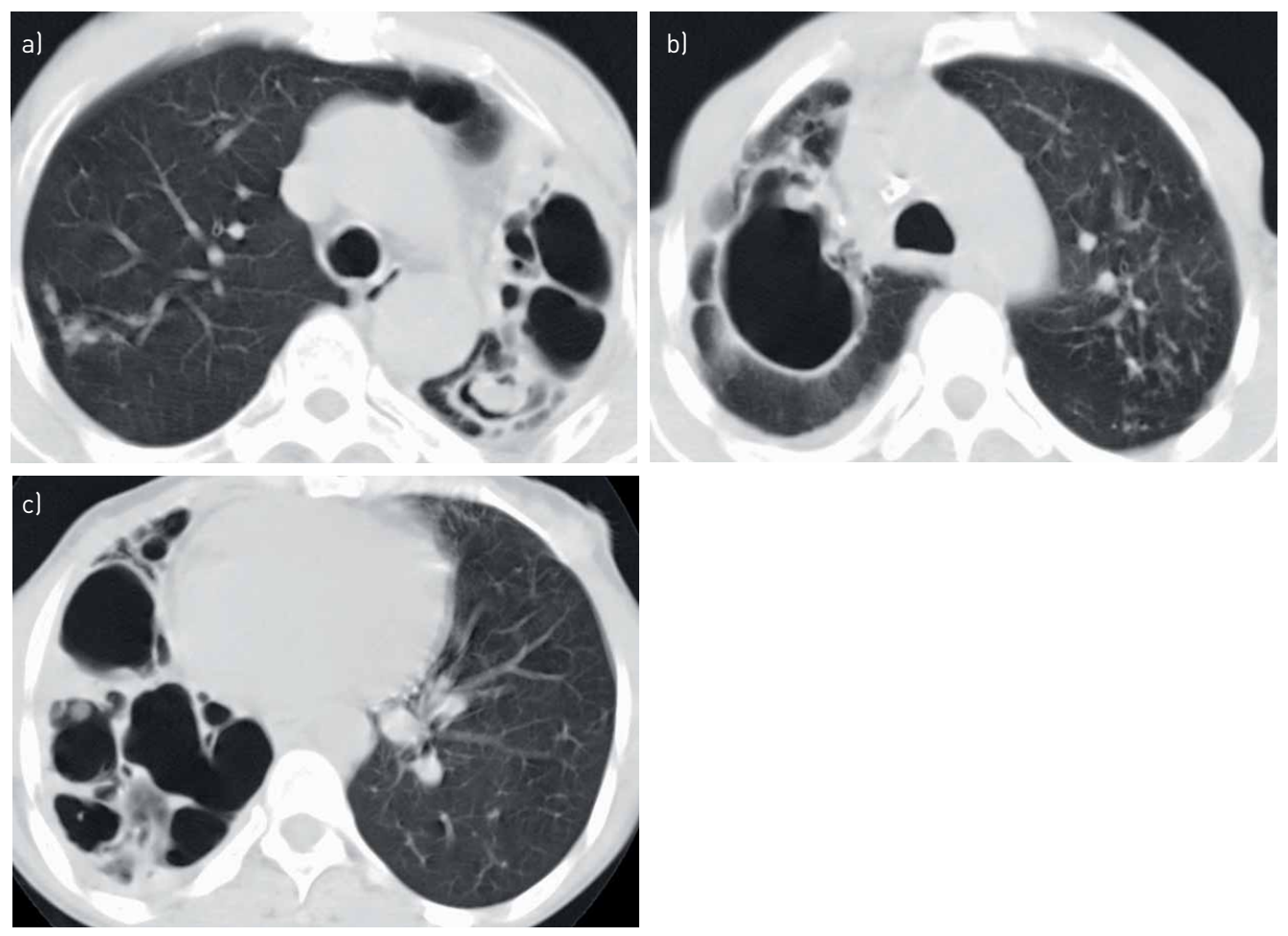

FIGURE 2 Radiological presentations of chronic pulmonary aspergillosis (CPA) in Gulu. a) Chronic cavitary pulmonary aspergillosis (CCPA) with multiple cavities and a fungal ball. b) CCPA with a large cavity, paracavitary fibrosis and adjacent pleural thickening, but no fungal ball. c) Chronic fibrosing pulmonary aspergillosis with complete destruction of the whole right lung with multiple cavities and surrounding fibrosis. 

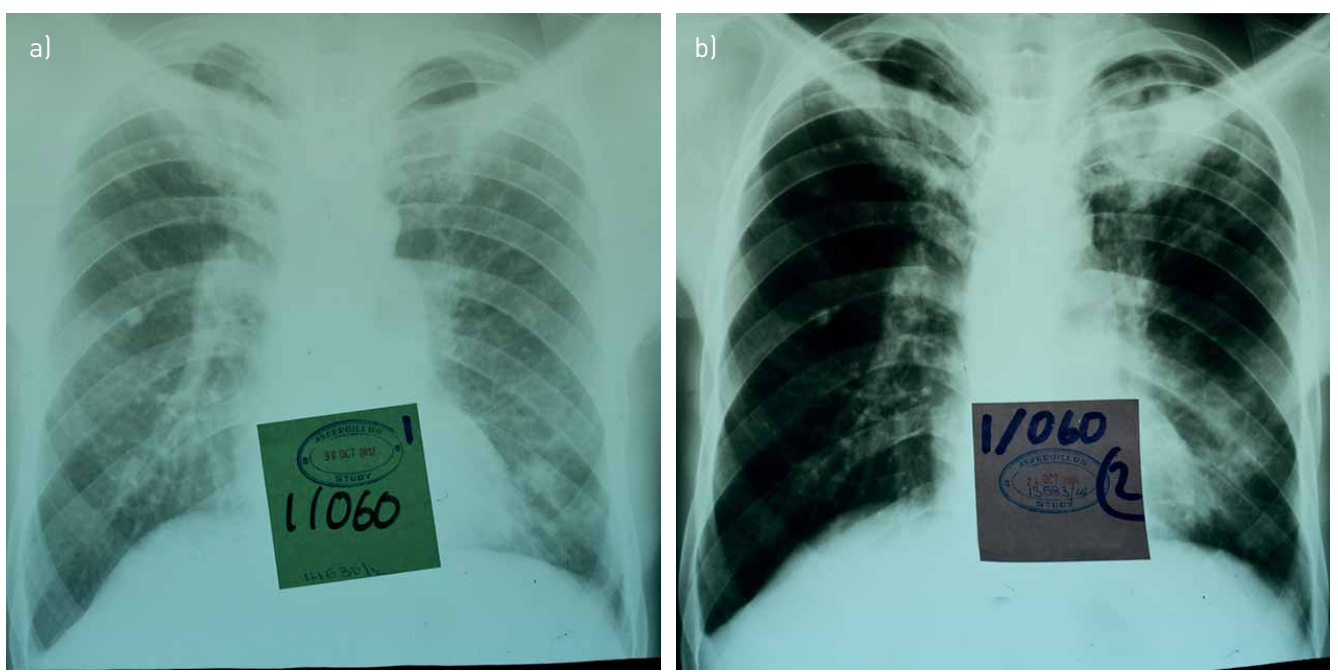

FIGURE 3 Chronic pulmonary aspergillosis (CPA) developing between surveys. a) Chest radiograph from December 2012 shows residual scarring at the left apex and a nodule at the left base in an HIV co-infected patient. b) Second radiograph from December 2014 shows a new cavity with a possible fungal ball at the left apex and evolution of the nodule into small thin-walled cavities at the left base, one probably with a small fungal ball. The patient also developed newly raised Aspergillus-specific IgG on resurvey. Computed tomography of the thorax was not performed as the patient had normal Aspergillus-specific IgG and no fungal ball on initial survey.

No associations were found between CPA diagnosis and potential clinical and radiological risk factors (supplementary table S2). Associations between individual symptoms and raised Aspergillus-specific IgG or $\mathrm{CPA}$ are shown in table 5. Cough and haemoptysis were significantly more common in those with raised Aspergillus-specific IgG, but other symptoms were not.

Fungal ball, cavitation, paracavitary fibrosis and pleural thickening were all significantly more common in those with raised Aspergillus-specific IgG, but bronchiectasis and nodules were not. Every patient with destroyed lung had raised Aspergillus-specific IgG and CPA. All forms of chest radiography progression were more common in CPA, except progressive cavitation.

The accuracy of combinations of symptoms and tests for CPA diagnosis is shown in table 6. All CPA cases had either cavitation or pleural thickening on chest radiography, but no combination of chest radiography findings and clinical assessment produced a positive predictive value $>40 \%$. Addition of Aspergillusspecific IgG measurement allowed CPA diagnosis with a positive predictive value of $92.3 \%$ and a negative predictive value of $99.3 \%$

\section{Discussion}

We have performed the first community-based survey of persons with treated pulmonary TB to measure CPA prevalence. It is the first CPA prevalence survey to achieve accurate diagnosis through systematic CT of the thorax and use of an Aspergillus-specific IgG assay validated for the study population.

The finding that CPA complicates $4.9-6.3 \%$ of all treated pulmonary TB cases and $26 \%$ of cases with chest radiography cavitation has profound implications for TB management programmes. It suggests that testing for CPA with Aspergillus-specific IgG should be routine in all patients with recurrent cough, haemoptysis or weight loss following treatment of pulmonary TB, regardless of HIV status or time from completion of TB therapy. Those with raised levels should have imaging, preferably with $\mathrm{CT}$ of the thorax, to confirm the diagnosis. As CPA mimics the presentation of pulmonary $\mathrm{TB}$, testing should also form part of routine investigations in any patient where empirical treatment for "smear-negative TB" is being considered.

Patients with chest radiography cavities had an annual rate of new CPA of at least 6.5\%, a condition associated with $38-80 \%$ 5-year mortality. This suggests that all patients should have chest radiography at the end of pulmonary TB treatment and that those with cavities should be monitored for CPA to facilitate early treatment. We identified new cases of CPA occurring up to 9 years after completion of TB therapy. The frequency of new CPA occurring beyond this point is unknown.

We have probably underestimated the frequency of CPA. 10 patients had a combination of symptoms and radiological features suggestive of CPA despite normal A. fumigatus-specific IgG, including two with a 
TABLE 5 Associations between Aspergillus and clinical and radiological findings

\section{All patients Aspergillus-specific Aspergillus-specific p-value OR $(95 \% \mathrm{CI}) \quad$ CPA present $\quad$ CPA absent p-value $\quad$ OR $(95 \% \mathrm{Cl})$}

\begin{tabular}{|c|c|c|c|c|c|c|c|c|c|}
\hline Symptoms & $n=285$ & $n=28$ & $n=257$ & & & $n=14$ & $n=271$ & & \\
\hline Cough $\#$ & $94(33.0)$ & $15(53.6)$ & 79 (30.7) & 0.015 & $2.60(1.18-5.72)$ & $11(78.6)$ & $83(30.6)$ & $<0.001$ & $8.30(2.26-30.55)$ \\
\hline Haemoptysis ${ }^{\#}$ & $21(7.4)$ & $8(28.6)$ & $13(5.1)$ & $<0.001$ & $7.51(2.78-20.24)$ & $7(50.0)$ & $14(5.2)$ & $<0.001$ & $18.36(5.65-59.60)$ \\
\hline Fatigue & 89 (31.2) & $9(32.1)$ & 80 (31.1) & 0.912 & $1.05(0.45-2.42)$ & $6(42.9)$ & $83(30.6)$ & 0.336 & $1.70(0.57-5.05)$ \\
\hline Fevers & $26(9.1)$ & $3(10.7)$ & $23(8.9)$ & 0.729 & $1.22(0.34-4.35)$ & $2(14.3)$ & $24(8.9)$ & 0.371 & $1.71(0.36-8.12)$ \\
\hline Night sweats & $51(17.9)$ & $6(21.4)$ & 45 (17.5) & 0.607 & $1.25(0.49-3.35)$ & $4(28.6)$ & 47 (17.3) & 0.287 & $1.91(0.57-6.34)$ \\
\hline Chest pain & $76(26.7)$ & 11 (39.3) & 65 (25.3) & 0.112 & $1.91(0.85-4.29)$ & $7(50.0)$ & $69(25.5)$ & 0.06 & $2.93(0.99-8.64)$ \\
\hline Chest radiography (resurvey) & $n=285$ & $n=28$ & $n=257$ & & & $n=14$ & $\mathrm{n}=271$ & & \\
\hline Cavitation & 50 (17.5) & $17(60.7)$ & 33 (12.8) & $<0.001$ & $10.49(4.52-23.34)$ & 12 (85.7) & $38(14.0)$ & $<0.001$ & 36.79 (7.92-170.87) \\
\hline Fungal ball & $13(4.6)$ & 5 (17.9) & $8(3.1)$ & $<0.001$ & $6.77(2.05-22.38)$ & $4(28.6)$ & 9 (3.3) & 0.002 & $11.643 .06-44.31$ \\
\hline Paracavitary fibrosis & $36(12.6)$ & $7(25.0)$ & 29 (11.3) & 0.038 & $2.62(1.02-6.7)$ & $4(28.6)$ & $32(11.8)$ & 0.085 & $2.99(0.88-10.09)$ \\
\hline Pleural thickening & 50 (17.5) & $16(57.1)$ & 34 (13.2) & $<0.001$ & 8.74 (3.81-20.07) & 10 (71.4) & $40(14.8)$ & $<0.001$ & $14.44(4.32-48.28)$ \\
\hline Chest radiography progression & $\mathrm{n}=285$ & $\mathrm{n}=28$ & $n=257$ & & & $n=14$ & $\mathrm{n}=271$ & & \\
\hline Cavitation & $7(2.5)$ & $4(14.3)$ & $3(1.2)$ & 0.002 & $14.06(2.97-66.51)$ & $1(7.1)$ & $6(2.2)$ & 0.301 & $3.38(0.38-30.21)$ \\
\hline Fungal ball & $7(2.5)$ & $3(10.7)$ & $4(1.6)$ & 0.023 & $7.56(1.60-35.70)$ & $2(14.3)$ & $5(1.8)$ & 0.041 & $8.83(1.55-50.27)$ \\
\hline Paracavitary fibrosis & 18 (6.3) & 5 (17.9) & $13(5.1)$ & 0.008 & $4.06(1.33-12.41)$ & $3(21.4)$ & $15(5.5)$ & 0.05 & $4.64(1.17-18.41)$ \\
\hline Pleural thickening & $16(5.6)$ & $5(17.9)$ & $11(4.3)$ & 0.003 & $4.84(1.55-15.14)$ & $3(21.4)$ & $13(4.8)$ & 0.037 & $5.39(1.34-21.71)$ \\
\hline Any progressive change & 34 (11.9) & $12(42.9)$ & $22(8.6)$ & $<0.001$ & 8.01 (3.37-19.06) & $7(50.0)$ & $27(10.0)$ & $<0.001$ & 9.04 (2.95-27.71) \\
\hline CT of the thorax & $\mathrm{n}=73$ & $n=21$ & $n=52$ & & & $n=14$ & $n=59$ & & \\
\hline Fungal ball ${ }^{\#}$ & $11(15.1)$ & $9(42.9)$ & $2(3.8)$ & $<0.001$ & 18.75 (3.58-98.28) & $9(64.3)$ & $2(3.4)$ & $<0.001$ & $51.3(8.61-305.47)$ \\
\hline Cavitation & 35 (47.9) & $16(76.2)$ & 19 (36.5) & 0.002 & $5.56(1.76-17.59)$ & $14(100)$ & $21(35.6)$ & $<0.001$ & \\
\hline Paracavitary fibrosis ${ }^{\#}$ & $28(38.4)$ & $16(76.2)$ & $12(23.1)$ & $<0.001$ & $10.67(3.23-35.19)$ & $14(100)$ & $14(23.7)$ & $<0.001$ & \\
\hline Pleural thickening & 48 (65.8) & 19 (90.5) & $29(55.8)$ & 0.005 & 7.53 (1.59-35.73) & $14(100)$ & $34(57.6)$ & 0.003 & \\
\hline Nodule(s) & $62(84.9)$ & $16(76.2)$ & 46 (88.5) & 0.185 & $0.42(0.11-1.56)$ & $10(71.4)$ & $52(88.1)$ & 0.116 & 0.337 (0.08-1.37) \\
\hline Bronchiectasis & $54(74.0)$ & $16(76.2)$ & $38(73.1)$ & 0.784 & $1.17(0.36-3.82)$ & $12(85.7)$ & $42(71.2)$ & 0.265 & $2.43(0.49-12.02)$ \\
\hline Destroyed lung & $3(4.1)$ & 3 (14.3) & 0 & 0.021 & & $3(21.4)$ & 0 & 0.006 & \\
\hline
\end{tabular}

Data are presented as $\mathrm{n}(\%)$, unless otherwise stated. CPA: chronic pulmonary aspergillosis; CT: computed tomography. \#: these features formed part of the CPA case definition. All $p$-values calculated by Fisher's exact test. Odds ratios were calculated by bimodal regression analysis. $p<0.05$ considered significant. 
TABLE 6 Accuracy of test combinations for chronic pulmonary aspergillosis (CPA) diagnosis in the absence of computed tomography of the thorax

\begin{tabular}{|c|c|c|c|c|c|}
\hline Test & $\mathbf{n}$ & Sensitivity \% & Specificity \% & PPV \% & NPV $\%$ \\
\hline Symptoms & 101 & 92.9 & 67.5 & 12.9 & 99.5 \\
\hline Chest radiography fungal ball & 13 & 28.6 & 96.7 & 30.8 & 96.3 \\
\hline Chest radiography cavitation & 50 & 85.7 & 86.0 & 24.0 & 99.1 \\
\hline Chest radiography pleural thickening & 50 & 71.4 & 85.2 & 20.0 & 98.3 \\
\hline Chest radiography cavitation or pleural thickening plus symptoms & 41 & 92.9 & 89.7 & 31.7 & 99.6 \\
\hline Serology plus chest radiography cavitation & 17 & 85.7 & 98.2 & 70.6 & 99.3 \\
\hline Serology plus chest radiography cavitation plus symptoms & 13 & 85.7 & 99.6 & 92.3 & 99.3 \\
\hline Serology plus chest radiography cavitation or pleural thickening plus symptoms & 17 & 92.3 & 98.5 & 76.5 & 99.6 \\
\hline
\end{tabular}

PPV: positive predictive value; NPV: negative predictive value. ${ }^{\#}$ : positive serology was a mandatory diagnostic criterion for CPA in this study.

fungal ball on CT of the thorax. These cases probably represent CPA cases caused by another species, as A. fumigatus-specific IgG may have low sensitivity in this group [17]. One patient also had HIV with a CD4 count $<200$ cells. $\mu \mathrm{L}^{-1}$. The accuracy of Aspergillus-specific IgG in this group is poorly described.

Our study has limitations. Convenience sampling allows recruitment bias, although this was minimised through active recruitment of eligible patients. Our case definition was written prior to the publication of the ERS guidelines [4]. These guidelines require either radiological or symptomatic evidence of disease duration $>3$ months for CCPA/CFPA. All our CCPA/CFPA patients met these criteria as 10 out of 13 (77.0\%) had symptoms for $>3$ months and three had chest radiography evidence of CPA in both surveys. The guidelines also require either a fungal ball on CT of the thorax or the combination of cavitation and radiological progression. 13 out of 14 (93\%) CPA cases met these criteria.

Subacute invasive pulmonary aspergillosis typically kills within a few months and is present in $2-11 \%$ of AIDS autopsies [28-32]. We used a 1-month symptom duration definition to allow inclusion of these patients, but we will not have identified patients who developed aspergillosis and died outside the survey periods.

Existing consensus case definitions for CPA are arbitrary and based on expert opinion. We have assessed associations between individual symptoms and raised Aspergillus-specific IgG and CPA for the first time. It may be appropriate to restrict future case definitions to cough and haemoptysis. Pleural thickening was present in most CPA cases and was significantly more common in those with CPA or raised Aspergillus-specific IgG. It may be appropriate to include CT of the thorax cavitation plus adjacent pleural thickening as radiological evidence of CPA in future case definitions. Progressive cavitation was the only form of chest radiography progression not associated with CPA diagnosis. Every patient with destroyed lung had raised Aspergillus-specific IgG and CPA, mandating investigations for CPA in this group.

Aspergillus colonisation of bronchiectatic airways is unlikely to be the main cause of raised Aspergillusspecific IgG in this population as there was no association between bronchiectasis and raised Aspergillus-specific IgG. The absence of any association between nodules and raised Aspergillus-specific IgG supports the current recommendation that biopsy is required to diagnose Aspergillus nodule disease $[4,33]$.

Prior studies demonstrate the presence of aspergillosis in other post-TB populations. The 1968-1970 study found aspergilloma in $20 \%$ of 544 British treated TB patients with residual cavities [27]. New Aspergillus-specific antibodies developed in $9.8 \%$ and aspergilloma in $5.4 \%$ of patients during 2 years of follow-up, indicating an annual rate of aspergilloma development of $2.7 \%$. Aspergillus-specific antibodies were found in $8.3 \%$ of 350 predominantly treated TB patients in Brazil [34], 23-25\% of 77 Indian treated TB patients [35, 36], 19\% of 226 Japanese patients with active or treated TB [37] and 9\% of 101 HIV-positive patients completing pulmonary TB treatment in Kampala [38]. Chest radiography plus serology surveys have found probable CPA in $8.7 \%$ of 208 patients completing TB therapy in Nigeria [22] and $11.3 \%$ of $124 \mathrm{HIV}$-negative patents with active or treated TB in Iran [23].

These prior studies are not directly comparable to ours as they took place in select clinical populations, used unvalidated or insensitive serology methods, lacked systematic CT imaging of the thorax and 
sometimes included non-TB patients in mixed cohorts. Nonetheless, aspergillosis was present at clinically important levels in every population studied and all the studies were consistent with CPA prevalence at least as high as our findings. The combination of our data and these prior studies demonstrates that CPA complicating pulmonary TB represents an unrecognised global public health issue.

Between 2000 and 2016, around 45 million lives were saved due to effective treatment of pulmonary TB worldwide [1]. However, 1.6-3.5 million of these would subsequently have developed CPA if this occurs within the $95 \%$ confidence interval identified in our study. Residual chest radiography cavitation is found in $20-30 \%$ of the 7.7 million patients cured of pulmonary TB each year [1]. If the annual rates of new CPA we found in those with and without residual cavitation are applied, then 112000-160000 people will develop CPA worldwide every year after completion of TB therapy.

This is probably an underestimate as we only diagnosed "new" CPA in those with negative baseline serology. Without baseline CT of the thorax we could not calculate the additional rate of "new" CPA in those with positive baseline serology but no radiological features. This estimate may also be low because it assumes that all patients with pulmonary TB actually had this disease and not CPA, and it omits any patients with both disorders concurrently who died during anti-TB therapy, with undiagnosed CPA.

Fungal ball is absent in most CPA cases [2]. Aspergillus-specific IgG and CT of the thorax are therefore required to reliably diagnose CPA cases. Unfortunately, both are rarely available in resource-poor settings [18]. In their absence patients with CPA meet WHO diagnostic criteria for "smear-negative TB" [39]. A recent Nigerian study found probable CPA in $19 \%$ of such patients, who were HIV-negative [22]. We found that a fungal ball on chest radiography was insensitive for diagnosing CPA. However, CPA could be excluded by chest radiography in this cohort and diagnosed with acceptable accuracy if Aspergillus-specific IgG measurement was also performed.

We therefore propose that CPA should be diagnosed in persons with treated TB in resource-poor settings if recurrent $\mathrm{TB}$ has been excluded by GeneXpert PCR testing and a combination of raised Aspergillus-specific IgG, chest radiography cavitation and either chronic cough or haemoptysis is present. This proposal requires validation in another population. Improved access to Aspergillus-specific IgG is necessary to diagnose CPA in resource-poor settings. Implementation of point-of-care tests, optimised for resource-poor settings, would facilitate this. A lateral flow device for Aspergillus-specific IgG has recently been commercialised in Europe (LDBio, Lyon, France).

Untreated CPA is usually fatal, but treatment is possible with relatively inexpensive oral generic antifungals, now included on the WHO Essential Medicine List [40], or surgery in selected cases. Meaningful improvements in TB-associated mortality are therefore possible by addressing CPA in a programmatic way within existing TB care systems. Public health bodies should now act to ensure equitable access to CPA diagnosis and treatment.

Acknowledgements: We would like to thank study assistants Patrick Kidega and Thomas Okumu (Gulu Regional Referral Hospital, Gulu, Uganda) for translation, phlebotomy and sample handling. Marion Surgenor, Helen Slattery and the Gulu Manchester Link team (Manchester University NHS Foundation Trust, Manchester, UK) for provision of accommodation in Gulu for study members and for logistical and practical assistance throughout the study. Staff at Gulu Regional Referral Hospital TB and HIV clinics for providing space to assess patients, identifying potential recruits, and facilitating sample storage and testing of sputum samples. All the staff at Gulu Regional District Health Office Tuberculosis and Leprosy Control department for identifying eligible patients and assisting in recruitment. Staff at the radiology departments at St Mary's Hospital, Lacor and Kampala Imaging Centre (Uganda) for their help with processing radiography and CT scans. Laboratory staff at Christie Hospital (Manchester, UK) for allowing access to their Siemens Immulite 2000 system to perform Aspergillus-specific IgG testing. Staff from Siemens for providing training on the Immulite 2000. Julie Morris (University of Manchester, Manchester, UK) for statistical support. University of Manchester students Andrew Mockridge, Matthew Kneale and Michael Clarke for their assistance with study set-up and recruitment.

Conflict of interest: I.D. Page reports grants from Astellas Pharma Europe, test kit donations from Siemens, grants and accommodation for study team from Gulu Manchester Link, during the conduct of the study; grants and test kit donation from Serion and Dynamiker, test kit donation from Genesis and OLM Medical, and travel grants for conference attendance from the British Infection Association, British Society for Medical Mycology, International Society for Human and Animal Mycology, European Society for Clinical Microbiology and Infectious Diseases, and Conference for Retroviruses and Opportunistic Infections, outside the submitted work. R. Byanyima reports grants from Astellas Pharma Europe, test kit donations from Siemens, grants and accommodation for study team from Gulu Manchester Link, during the conduct of the study. S. Hosmane reports grants from Astellas Pharma Europe, test kit donations from Siemens, grants and accommodation for study team from Gulu Manchester Link, during the conduct of the study. N. Onyachi reports grants from Astellas Pharma Europe, test kit donations from Siemens, grants and accommodation for study team from Gulu Manchester Link, during the conduct of the study. C. Opira reports grants from Astellas Pharma Europe, test kit donations from Siemens, grants and accommodation for study team from Gulu Manchester Link, during the conduct of the study. M. Richardson reports grants from Astellas Pharma Europe, test kit donations from Siemens, grants and accommodation for study team from Gulu Manchester Link, during the conduct of 
the study; personal fees for talks and consultancy from Gilead Sciences Europe, MSD and Basilea, outside the submitted work. R. Sawyer reports grants from Astellas Pharma Europe, test kit donations from Siemens, grants and accommodation for study team from Gulu Manchester Link, during the conduct of the study. A. Sharman reports grants from Astellas Pharma Europe, test kit donations from Siemens, grants and accommodation for study team from Gulu Manchester Link, during the conduct of the study. D.W. Denning reports grants from Astellas Pharma Europe, test kit donations from Siemens, grants and accommodation for study team from Gulu Manchester Link, during the conduct of the study; has founder shares with F2G, has received personal fees for consultancy from Scynexis, Cidara, Biosergen, Quintiles, Pulmatrix, Pulmocide, Zambon and Fujifilm, personal fees for lectures from Astellas, Dynamiker, Gilead, Merck and Pfizer, outside the submitted work; and is a long-standing member of the Infectious Disease Society of America Aspergillosis Guidelines group, the European Society for Clinical Microbiology and Infectious Diseases Aspergillosis Guidelines group, and the British Society for Medical Mycology Standards of Care committee.

Support statement: The study was funded by an investigator-driven study grant from Astellas Pharma Europe Ltd. Further financial support and accommodation for study members in Gulu was provided by the Gulu Manchester Link charity, based at Wythenshawe Hospital (Manchester, UK). Aspergillus-specific IgG test kits used in the study were donated by Siemens. No funder had any role in study design or interpretation of results. Funding information for this article has been deposited with the Crossref Funder Registry.

\section{References}

1 World Health Organization. WHO Global Tuberculosis Report. 2017. www.who.int/tb/publications/global_report/ en Date last accessed: January 8, 2018.

2 Jhun BW, Jeon K, Eom JS, et al. Clinical characteristics and treatment outcomes of chronic pulmonary aspergillosis. Med Mycol 2013; 51: 811-817.

3 Ohba H, Miwa S, Shirai M, et al. Clinical characteristics and prognosis of chronic pulmonary aspergillosis. Respir Med 2012; 106: 724-729.

4 Denning DW, Cadranel J, Beigelman-Aubry C, et al. Chronic pulmonary aspergillosis: rationale and clinical guidelines for diagnosis and management. Eur Respir J 2016; 47: 45-68.

5 Page ID, Richardson MD, Denning DW. Comparison of six Aspergillus-specific IgG assays for the diagnosis of chronic pulmonary aspergillosis (CPA). J Infect 2016; 72: 240-249.

6 Smith NL, Denning DW. Underlying conditions in chronic pulmonary aspergillosis including simple aspergilloma. Eur Respir J 2011; 37: 865-872.

7 Lowes D, Al-Shair K, Newton PJ, et al. Predictors of mortality in chronic pulmonary aspergillosis. Eur Respir J 2017; 49: 1601062 .

8 Chen Q-K, Jiang G-N, Ding J-A. Surgical treatment for pulmonary aspergilloma: a 35-year experience in the Chinese population. Interact Cardiovasc Thorac Surg 2012; 15: 77-80.

9 Farid S, Mohamed S, Devbhandari M, et al. Results of surgery for chronic pulmonary aspergillosis, optimal antifungal therapy and proposed high risk factors for recurrence - a national centre's experience. $J$ Cardiothorac Surg 2013; 8: 180 .

10 Ba M, Ciss G, Diarra O, et al. Aspects chirurgicaux des aspergillomes a propos de 24 malades. [Surgical aspects of pulmonary aspergilloma in 24 patients.] Dakar Med 2000; 45: 144-146.

11 Ngo Nonga B, Bang GA, Jemea B, et al. Complex pulmonary aspergilloma: surgical challenges in a Third World setting. Surg Res Pract 2018; 2018: 6570741.

12 Agarwal R, Vishwanath G, Aggarwal AN, et al. Itraconazole in chronic cavitary pulmonary aspergillosis: a randomised controlled trial and systematic review of literature. Mycoses 2013; 56: 559-570.

13 Al-Shair K, Atherton GT, Harris C, et al. Long-term antifungal treatment improves health status in patients with chronic pulmonary aspergillosis: a longitudinal analysis. Clin Infect Dis 2013; 57: 828-835.

14 Camuset J, Nunes H, Dombret M-C, et al. Treatment of chronic pulmonary aspergillosis by voriconazole in nonimmunocompromised patients. Chest 2007; 131: 1435-1441.

15 Kosmidis C, Newton P, Muldoon EG, et al. Chronic fibrosing pulmonary aspergillosis: a cause of "destroyed lung" syndrome. Infect Dis 2017; 49: 296-301.

16 Patterson TF, Thompson GR, Denning DW, et al. Practice guidelines for the diagnosis and management of aspergillosis: 2016 update by the Infectious Diseases Society of America. Clin Infect Dis 2016; 63: e1-e60.

17 Page ID, Richardson M, Denning DW. Antibody testing in aspergillosis - quo vadis? Med Mycol 2015; 53: 417-439.

18 Cole DC, Govender NP, Chakrabarti A, et al. Improvement of fungal disease identification and management: combined health systems and public health approaches. Lancet Infect Dis 2017; 17: e412-e419.

19 Page ID, Richardson MD, Denning DW. Siemens Immulite Aspergillus-specific IgG assay for chronic pulmonary aspergillosis diagnosis. Med Mycol 2019; 57: 300-307.

20 Denning D, Pleuvry A, Cole D. Global burden of chronic pulmonary aspergillosis as a sequel to pulmonary tuberculosis. Bull World Health Organ 2011; 89: 864-872.

21 Denning DW, Riniotis K, Dobrashian R, et al. Chronic cavitary and fibrosing pulmonary and pleural aspergillosis: case series, proposed nomenclature change, and review. Clin Infect Dis 2003; 37: Suppl. 3, S265-S280.

22 Oladele RO, Irurhe NK, Foden P, et al. Chronic pulmonary aspergillosis as a cause of smear-negative TB and/or TB treatment failure in Nigerians. Int J Tuberc Lung Dis 2017; 21: 1056-1061.

23 Hedayati MT, Azimi Y, Droudinia A, et al. Prevalence of chronic pulmonary aspergillosis in patients with tuberculosis from Iran. Eur J Clin Microbiol Infect Dis 2015; 34: 1759-1765.

24 Tiendrebeogo H, Sangare SI, Roudaut M, et al. Cent un cas d'aspergillose pulmonaire en Cote-d'Ivoire. [One hundred and one cases of pulmonary aspergillosis in Ivory Coast.] Med Trop 1982; 42: 47-52.

25 Meawed TE, Fawzy MA, El Gamry RM, et al. Frequency of pulmonary fungal infection in Egyptian patients with re-treatment pulmonary tuberculosis and its clinical and radiological significance. J Am Sci 2012; 8: 683-691.

26 Adebonojo SA, Osinowo O, Adebo O. Lung abscess: a review of three-years' experience at the University College Hospital, Ibadan. J Natl Med Assoc 1979; 71: 39-43. 
27 British Tuberculosis Association. Aspergilloma and residual tuberculous cavities - the results of a resurvey. Tubercle 1970; 51: 227-245.

28 Denning DW, Follansbee SE, Scolaro M, et al. Pulmonary aspergillosis in the acquired immunodeficiency syndrome. N Engl J Med 1991; 324: 654-662.

29 Antinori S, Nebuloni M, Magni C, et al. Trends in the postmortem diagnosis of opportunistic invasive fungal infections in patients with AIDS: a retrospective study of 1630 autopsies performed between 1984 and 2002. Am J Clin Pathol 2009; 132: 221-227.

30 Lanjewar DN, Duggal R. Pulmonary pathology in patients with AIDS: an autopsy study from Mumbai. HIV Med 2001; 2: 266-271.

31 Katano H, Hishima T, Mochizuki M, et al. The prevalence of opportunistic infections and malignancies in autopsied patients with human immunodeficiency virus infection in Japan. BMC Infect Dis 2014; 14: 229.

32 Cox J, Lukande R. Needle autopsy to establish the cause of death in HIV-infected hospitalized adults in Uganda: a comparison to complete autopsy. I Acquir Immune Defic Syndr 2014; 67: 169-176.

33 Muldoon EG, Sharman A, Page I, et al. Aspergillus nodules; another presentation of chronic pulmonary aspergillosis. BMC Pulm Med 2016; 16: 123.

34 Ferreira-Da-Cruz MF, Wanke B, Pirmez C, et al. Aspergillus fumigatus fungus ball in hospitalized patients with chronic pulmonary disease. Usefulness of double immunodiffusion test as a screening procedure. Mem Inst Oswaldo Cruz 1988; 83: 357-360.

35 Shahid M, Malik A, Bhargava R. Prevalence of aspergillosis in chronic lung diseases. Indian J Med Microbiol 2001; 19: 201-205.

36 Kurhade AM, Deshmukh JM, Fule RP, et al. Mycological and serological study of pulmonary aspergillosis in central India. Indian J Med Microbiol 2002; 20: 141-144.

37 Iwata H, Miwa T, Takagi K. [Pulmonary tuberculosis and mycotic infection - clinical and serological diagnosis.] Kekkaku 1989; 64: 7-13.

38 Kwizera R, Parkes-Ratanshi R, Page ID, et al. Elevated Aspergillus-specific antibody levels among HIV infected Ugandans with pulmonary tuberculosis. BMC Pulm Med 2017; 17: 149.

39 World Health Organization. Improving the diagnosis and treatment of smear-negative pulmonary and extrapulmonary tuberculosis among adults and adolescents. Recommendations for HIV-prevalent and resource-constrained settings. 2006. www.who.int/tb/publications/2006/tbhiv_recommendations.pdf Date last accessed: February 15, 2018.

40 World Health Organization. WHO Model List of Essential Medicines. 2017. www.who.int/medicines/publications/ essentialmedicines/20th_EML2017.pdf Date last accessed: February 14, 2018. 